\title{
Théodicée dans l'Ancien Testament
}

\author{
par \\ MASAO SEKINE \\ professeur à l'Université de l'Éducation de Tokio
}

Peut-on reconnaître la théodicée dans l'Ancien Testament? Si l'on le peut, quelle est-elle? Jusqu'ici, la question n'a reçu aucune réponse décisive. Pour $\mathbf{y}$ répondre, il faut d'abord distinguer les deux aspects qu'elle comporte. Premièrement, c'est une question de terminologie: qu'est-ce qu'on entend par le mot théodicée? Deuxièmement, c'est une question plus essentielle qui concerne le fond: comment faut-il comprendre les faits décrits dans l'Ancien Testament?

Le mot théodicée est très souvent utilisé dans les études critiques de l'Ancien Testament. W. Staerk définit le mot, dès le début de son livre "Vorsehung und Vergeltung. Zur Frage nach der sittlichen Weltordnung 1931: "Theodizee, d.h. Rechtfertigung der göttlichen Weltleitung, nennt man seit Leibniz die theoretische Bemühung, den in aller lebendigen Religion empfundenen Widerspruch zwischen dem Sinn der Welt, den der sittliche Gottesgedanke behauptet, und dem tatsächlichen Weltverlauf $\mathrm{zu}$ beseitigen. Theodizee ist also Rechtfertigung Gottes im Sinne von Pslm 51,6 und Röm 3,4." On doit se demander ce qu'il entend par "theoretisch" quand il dit "theoretische Bemühung." Si cela signifie la théorie dans l'acception rigoureusement philosophique du mot, c'est-à-dire, "la théorie, pure et abstraite," "la théorie pour la théorie," il n'y a de théodicée ni dans l'Ancien Testament, ni dans aucune de ce qu'il appelle "la religion vivante." En réalité Staerk admet dans l'Ancien Testament des tentatives pour la théodicée, mais il insiste sur l'importance de comprendre cette théodicée plus par la foi que par la théodicée explicite. Selon lui, par exemple, la question posée par le Livre de Job est résolue à la fin, quand Job s'aperçoit de son erreur d'avoir proposé la théodicée. ${ }^{(1)}$ W. Eichrodt pousse plus loin cette opinion de Staerk. Il va jusqu'à nier l'existence de théodicée dans l'Ancien Testament, car il considère la théodicée comme arguments purement spéculatifs. Par exemple, il dit.

(1) Staerk, o. c. p. 44 
qu'il faut voir la foi d'Ezéchiel, et non sa théodicée, dans ses arguments sur la responsabilité personelle, Ez. 18, partie où précisément Staerk trouve la notion abstraite de la théodicée. ${ }^{(2)}$ Dans ce cas, la question n'est pas seulement de constater qu'Eichrodt prend le mot théodicée dans en sens trop étroit, mais de savoir comment il interprète les faits communiqués dans l'Ancien Testament. Je pense comme Eichrodt qu'il n'y a pas de notion abstraite en Ez. 18, mais je ne pense pas qu'il soit juste d'y voir seulement la foi d'Ezechiel. Sa manière de raisonner en divisant la question en parcelles et les résolvant l'une après l'autre nous oblige à y reconnaître certains efforts logiques, -nous donnons au mot le sens étymologique de $\lambda o ́ r o s . ~ E s t-c e$ que la foi et l'effort logique sont, comme on le croit, deux choses absolument incompatibles dans l'Ancien Testament? C'est très discutable. Si nous pensions que la solution de Job consiste à ce que Job répond par la foi à la révélation de Dieu, quand il l'a à la fin, nous pourrions dire que la question de foi, et non celle de théodicée, fait le centre du Livre de Job. Mais il faut d'abord se demander s'il est juste d'interpréter Job de cette façon. Pour moi, je pense qu'à travers tout le Livre de Job, la structure logique est très sensible: dans l'épiphanie, décrite aux ch. 38 ss,-plus précisément dans 38,4 et 40,15 - on peut trouver les arguments qui correspondent aux chapitres précédents. ${ }^{(3)}$ Si l'on interprète tout simplement que Job se prosterne devant l'épiphanie ou la révélation de Dieu, cela n'a aucune relation essentielle avec ce qui précède. Par conséquent, la question n'est pas seulement que les critiques ont pris le mot théodicée dans un sens trop étroit, mais qu'ils ont envisagé les textes de l'Ancien Testament uniquement de point de vue de la foi. Je tâcherai donc de prouver ci-dessous que dans l'Ancien Testament, il y a plus d'efforts logiques que l'on imaginait.

Avant d'entrer en matière, je voudrais donner mon opinion personelle sur celles des autres critiques. Si Staerk et Eichrodt prennent le mot théodicée dans en sens trop étroit, en l'opposant diametralement à la foi, Max Weber le prend dans un sens trop large. La théodicée lui sert d'un des critères importants, quand il compare la "ethique économique" des religions du monde dans sa "Religionssoziologie." Selon lui, parmi toutes les religions du monde, il y

(2) Eichrodt, Vorsehungsglaube und Theodizee im Alten Testament (Festschrift Procksch 1934) p. 60 ss; Staerk, o. c. p. 36.

(3) Sekine, Schöpfung und Erlösung im Buche Hiob (Festschrift Eissfeldt 1958) 
en a trois qui revêtent des formes apparentes de théodicée : d'abord le dualisme de la religion perse, deuxièmement, le dogme du karma de la religion des Indes, troisièmement la prédestination du calvinisme. ${ }^{(4)}$ Il voit par exemple dans la systématisation des magies en Babylonie un motif de théodicée, ou encore, dans l'idée de jugement chez les prophètes israélites, la "théodicée de malheur." (j) Il dit qu'en Babylonie, on admettait des démons, en même temps que dieu, et imputant à ceux-la l'existence du mal, rendait possible la confiance en dieu. Mais dans ce dualisme, il s'agit avant tout de savoir si on peut parler de la théodicée dans le sens rigoureux du mot, car, peut-on concevoir la théodicée sans supposer un seul créateur, un seul dominateur du monde? Si l'on ne peut, n'est-il pas impossible, par la même raison, que le dogme du karma puisse engendrer une théodicée, puisque l'immense mécanisme de causalité de ce dogme ne peut comporter un seul Dieu? Dans le cas des prophètes israélites, leur idée de jugement est certes la réponse, de la part de Dieu, à la question du mal dans le monde, en particulier en Inraël; mais l'idée de jugement chez Weber a-t-elle quelque chose d'efforts logiques? S'il est juste, comme nous le croyons, de supposer des efforts logiques dans la théodicée, il faut dire que Weber donne un sens trop large à ce mot, en disant "Unheilstheodizee." Il lui serait permis d'employer ce mot dans ce sens, quand, suivant sa méthode sociologique, il fait la comparaison des éthiques économiques des religions, mais au point de vue des études de l'Ancien Testament il faut faire entrer dans le mot théodicée une certaine idée d'efforts logiques, sinon théoriques, pour supprimer la contradiction entre l'existence de Dieu - en supposant un seul Dieu - et celle du mal.

Nous entrons ici dans le vif de la question. Partons de l'idée de jugement chez les prophètes: car, nous y trouvons une théodicée, différente de celle que Weber a reconnue, puisque nous y voyons des efforts logiques.

Dans l'histoire de l'idée de jugement, Amos, prophète du VIIIe siècle, fait époque; c'est de lui que date l'idée de jugement au sens précis du mot. A. Weiser étudie soigneusement dans son ouvre "Die Prophetie des Amos" 1929 le processus de la formation de la prophétie chez Amos, et constate que la particularité de sa prophétie consiste dans l'étroite union de l'expérience

(4) Weber, Wirtschaft und Gesellschaft 1925 II Teil. IV Kap. §8 Das Problem der Theodizee; Gesammelte Aufsätze zur Religionssoziologie I. 1922, p. 241 ss. 571 ss.

(5) Weber, Gesammelte Aufsätze zur Religionssoziologie III 1923, p. 229 ss. 237. 314 ss. 328 ss. 
irrationnelle et de la pensée rationelle. Il faut faire attention que Weiser insiste sur l'élément rationnel, c'est-à-dire la pensée. Mais, en ce qui concerne l'idée de jugement, l'explication de Weiser nous parait peu satisfaisante. Il dit que cette idée fait le noyau de la prophétie d'Amos. Je suis d'accord sur ce point. Mais il fait parvenir cette idée de l'expérience religieuse très particulière du prophète et ne dit rien de précis de l'opération intellectuelle de son esprit. Il dit: "Der Gedanke der inneren Notwendigkeit des Gerichts steht dem Amos so fest, dass er direkt zur logischen Norm wird." "Da nun gerade in dem, den Visionen zugrundeliegenden Erleben die ethischrechtliche Färbung des Gerichtsbegriffs fehlt, die Gerichtsgewissheit dagegen besteht, so ist die Annahme nicht von der Hand zu weisen, dass der Gerichtsgedanke unmittelbar aus der Eigenart des Gotteserlebens hervorgegangen ist." (6) Mais l'expérience ne peut pas devenir directment idée. Pour qu'elle le divienne, il faut des efforts rationnels de l'esprit. Il en est de même de l'idée de jugement, qui est un "norme logique." A ce sujet, Am. 3,2 et 9,7 me paraissent particulièrement importants :

3,2 Je n'ai connu que vous de toute les familles de la terre, aussi vous visiterai-je pour toute vos iniquités.

9,7 N'êtes-vous pas pour moi comme des Kushite, enfants d'Israël? oracle de Yahvé. N'ai-je pas fait monter Israël du pays d'Egypte, comme les Philistins de Kaphtor, et les Araméens de Qir? (tr. E. Osty) Au premier coup d'œil, ces deux textes paraissent se contredire: selon l'un, Dieu ne connait qu'Israël; selon l'autre, il le considère comme un peuple parmi beaucoup d'autres. Il y en a donc qui nient l'un des deux, ou bien, comme Rieger, qui y voient la coexistence des deux dieux contradictoires l'un avec l'autre, du dieu dominateur du monde et du dieu national d'un peuple. ${ }^{(7)}$ L'interprétation de Weiser sur ce point attire notre attention. Selon lui, 3,2 cite fidèlement la protestation du peuple à l'égard de la prophétie d'Amos, et Amos lui même, n'en parle que pour faire ressortir sá propre conclusion: "visitrai-je." $\mathrm{Si}$ l'on adopte cette interprétation, la contradiction alleguée ci-dessus disparaîtrait: car la pensée d'Amos serait parfaitement cohérente, en ce qu'il considère Israël comme un peuple parmi beaucoup

(6) Weiser, o.c. p. 311.

(7) Rieger, Die Bedeutung der Geschichte für die Verkündigung des Amos und Hosea 1929, pp. 30. 36. 95 . 
d'autres. ${ }^{(8)}$ Cependant, cette interprétation est inacceptable : car, même s'il était vrai que les paroles du peuple étaient citées mot, à mot, il est impossible. de penser, comme Weiser, qu'Amos ne leur accorde aucune importance; d'où. qu'elles viennent, puisqu'il les a citées, sa conclusion doit s'appuyer sur elles. Selon moi, l'apparente contradiction de ces deux textes, 3,2 et 9,7, montre au contraire le logique de l'idée de jugement sur arrière-plan d'élection chez Amos, par conséquent, la logique de sa théodicée.

H. H. Rowley et A. Neher traduisent cette expression de Am. 3,2: “ je n'ai connu que vous" par le mot elect, choisir. $^{(9)}$ Th. C. Vriezen ne veut y voir que la relation intérieure de Dieu et d'Israël. ${ }^{(10)}$ V. Maag dit, que "jadha' 'ethkhem» avec «raḳ» exprime la notion d'élection. ${ }^{(11)}$ La question est ici de décider s'il y a ou non l'idée d'élection dans cette expression. Ce n'est pas une simple question de terminologie. C'est la structure de la pensée qui importe. La recherche de Rowley, ni celle de Vriezen sur l'idée d'élection n'en tiennent compte.

Je pense que les critiques ont en tort d'identifier l'idée d'élection avec celle d'alliance. L'alliance est une affaire entre Dieu et Israël, tandis que l'élection signifie: choisir un parmi plusieurs. Par conséquent, l'idée d'élection doit partir de la connaissance qu'Israël est un peuple parmi d'autres. ${ }^{(12)}$ Et justement, 9,7 montre cette connaissance. En ce sens 9,7 peut être considéré comme préambule pour arriver à 3,2 . Israël est aux yeux de Dieu un peuple parmi beaucoup d'autres. Cela permet à Dieu de le choisir selon son: gré et surtout, de le punir à cause de ses péchés. ${ }^{(13)}$

Ainsi, 3,2 et 9,7 ne sont pas liés d'une logique superficielle, mais d'une

(8) Weiser, o. c. p. 120 ss.

(9) Rowley, The Biblical Doctrine of Election 1950, p. 53; Neher, Amos. Contribution a l'étude: du prophétisme 1950 , p. 34 ss.

(10) Vriezen, Die Erwählung Israëls nach dem Alten Testament 1953, p. 37.

(11) Maag, Text, Wortschatz und Begriffswelt des Buches Amos 1951, p.152.

(12) W. Eichrodt, Gottes Ruf im Alten Testament 1951 p. 36 suggère avec justesse le problème. de "Gegensatz zwischen Einheit und Vielheit" à l'égard de Gen. 12, 1-3.

(13) L'interprétation de Neher dans son livre déjà cit乏 (p. 48) est relativement proche de la nôtre. Il dit: "L'élection d'Israël ne se justifie (historiquement et mátaphysiquement) que sur l'arrière-plan de l'élection universelle (création). Ici s'établit le pont entre les versets III, 1-2 et IX, 7 d'Amos, ce pont que l'exégèse wellhausenienne n'a pu apercevoir parce qu'elle méconnaissait la valeur de la notion de berith." Seulement Neher ne distingue pas entre l'élection et l'alliance. N'oublions pas que la relation de $3,2 \mathrm{a}$ et $3,2 \mathrm{~b}$ montre que chez Amos, l’idée de jugement est née de celle d'élection. 
logique profondément intérieure. Pour insister sur ce point, je voudrais ajouter encore quelque mots. Avant Amos, et surtout dans la foi populaire, Dieu et Israël étaient trop intimement liés pour être indépendants l'un de l'autre. Dans ces conditions, il était difficile que Dieu punisse Israël. Chez Amos Dieu s'est libéré d'Israël, et par la même, devenu capable de la choisir et, capable pour la première fois, de le punir. En même temps qu'Israël est devenu un peuple parmi d'autres, c'est-à-dire, un “particulier," Dieu national d'un peuple s'est transformé en Dieu universel. C'est cette structure de l'idée de jugement qui garantit l'indépendance de Dieu à l'égard des malheurs de ses adhérants et de leur péchés, et qui par là même, ouvre le chemin de la " théodicée de malheur."

Dans la prophétie d'Amos, il ne s'agit d'un bout à l'autre que de l'idée de jugement, c'est-à-dire, quelque chose de négatif. Mais, chez les prophètes après Amos, cette idée de jugement est dépassée par celle de salut. La restauration universelle d'Osée (Os. 2, $14 \mathrm{ss}$ ), le venir du Messie d'Isaïe (Is. 9. 11. 16,1-5), la nouvelle alliance de Jérémie (Jer. 31, 31 ss), l'oracle du Serviteur de Yahvé du Second Isaïe (Is. 53), toutes ces idées appartiennent à l'eschatologie, née de la médiation négative par l'individuel entre le particulier et l'universel. Nous pensons que ce qui constitue le centre de la théodicée chez les prophètes est l'eschatologie qui contient en elle des efforts logiques. En cela précisément l'eschatologie diffère de la vague espérence de l'avenir. Récemment, J. Lindblom et Th. C. Vriezen ont essayé de savoir s'il y a l'eschatologie dans l'Ancien Testament. ${ }^{(14)}$ Malheureusement, leurs études se bornent au fond à la terminologie et ne vont pas jusqu'à l'éclaircissement de la structure logique de la prophétie.

Pour porter plus de lumière sur cette question, prenons Jer. 12, 1-5. Il est vrai que 12, 1-6 nous sont transmis en bloc, mais je voudrais, avec d'autres commentateurs, apporter les vv. $3 \mathrm{~b}, 6$ au ch. 11 et v. $4 \mathrm{~b}$ après v. 2 :

12, $1 \mathrm{Tu}$ as le droit pour toi, Yahvé, quand je prétends requérir contre toi.

Je voudrais seulement débattre avec toi un point de justice :

Pourquoi le sort des méchants est-il prospère?

(14) Lindblom, Gibt es eine Eschatologie bei den alttestamentlichen Propheten? (Studia Theologica vol. VI. p. $79 \mathrm{ss}$ ); Vriezen, Prophecy and Eschatology (Supplements to Vetus Testamentum vol. I p. 197 ss.) 
Pourquoi tous les perfides goûtent-ils la paix ?

2 Tu les plantes; ils s'enracinent: ils viennent bien : ils portent du fruit.

Pourtant tu n'es près que de leur bouche et loin de leurs reins.

4b Car ils disent: Dieu ne voit pas nos chemins.

3a Mais toi, Yahvé, tu me connais, tu me vois: tu éprouves mon cceur : il est avec toi.

4a Jusques à quand le pays sera-t-il en deuil et l'herbe de tout la campagne desséchée?

C'est par la perversité de ses habitants que périssent bétail et oiseaux.

5 Si cela te fatigue de faire la course avec des piétons, comment te mesureras-tu aux chevaux?

Et si dans un pay tranquille tu n'es pas sûr, que feras-tu dans les halliers du Jourdain ?(15) (tr. A. Gelin)

Ce passage est en géneral considéré comme une expression typique de la théodicée de Jérémie: Jérémie propose ici la question de théodicée et Dieu donne v. 5 comme réponse: ce que veut dire v. 5 est, selon Staerk, "Frage nicht, sondern glaube."(16) Eichrodt pense comme cela dans la monographie déjà citée. ${ }^{(17)}$ Weiser, dans la partie de son commentaire qui regarde ce passage, pousse cette opinion plus loin jusqu'à dire que Dieu demande ici à Jérémie "sacrificium intellectus" et la " confiance aveugle."(18) Nous ne pensons pas que Dieu soit capable de demander pareille chose. En tout cas, il ne nous paraît pas juste de penser, comme eux, que la foi n'a aucune affaire avec la logique. Etudions avant tout le text avec soin. On pense ordinairement que Jérémie ici a d'abord eu la conscience individuelle, et parvient de là à révoquer devant Dieu le bonheur des méchants en doute. Mais c'est une mauvaise interprétation que d'arracher Jérémie à son milieu, à Israël, et de lui faire parler en qualité d'individu indépendant du peuple. D'une part, Jérémie se considère comme partie d'un tout: il souffre à cause précisément

(15) Selon la tradiction proposé par G.R. Driver, "Difficult Words in the Hebrew Prophets." dans "Studies in Old Testament Prophecy presented to T. H. Robinson" 1950 p. 59 : "and if thou fallest flat on thy belly in a land of peace, how then willt thou fare in the risin: surge of the stream ?" Le sens d'ensemble ne change guère

(16) Staerk o.c. p. 31

(17) Eichrodt, o.c. p. 61

-(18) Weiser, Der Prophet Jeremia 1952-55, ad loc. 
de cela. D'autre part, v. 2 montre que les méchants en question sont ceux d'Israël, peuple d'élection. Il n'est pas donc vrai que Jérémie propose la question de théodicée, en se plaçant hors d'Israël. Tout au contraire, il se situe au dedans et s'impose la question comme une question existentielle. Ceci explique une contradiction qui se trouve au v. 1; “ $\mathrm{Tu}$ as le droit pour toi, Yahvé, quand je prétends requérir contre toi." En effet, dans les textes cités en haut, nous pouvons trouver le motif pour nier l'ancien Israël, mais il reste toujours vrai que la question est dans l'intérieur d'Israël. On n'a donc pas à supprimer v. $4 \mathrm{~b}$, comme le font beaucoup de commentateurs. W. Rudolph par exemple dit: quand il s'agit du bonheur des méchants, on n'a pas besoin de tenir compte des maux généraux qui tombent sur les dévots comme sur les méchants. Il faut donc supprimer ces lignes. ${ }^{(19)}$ Cette remarque trahit le défaut de l'interprétation traditionelle du passage en question. Il serait juste de distinguer nettement entre les dévots et les méchants au point de vue du judaïsme, qui considère que tout les méchants sont hors du cercle dévot. Mais Jérémie met en question Israël tout entier, y compris les méchants : si la Terre promise est dévastée, c'est à cause des péchés du peuple. v. $4 \mathrm{a}$ vient à l'appui de notre interprétation.

On considère généralement que v. 5 est la réponse de Dieu à Jérémie et que les "piétons" sont opposés aux "chevaux," le "pays tranquille" aux “halliers du Jourdain." Le texte massorétique rapprochant v. 6 à v. 5 , insiste que la persécution exercée sur Jérémie par sa propre famille lui serait plus pénible que la persécution générale. ${ }^{(20)}$ Mais on ne peut pas considérer comme "pays tranquille" celui où Jérémie a failli être assassiné. D'ailleurs, si séparant v. 6 de v. 5 , on l'ajoute au ch. 11, cette interprétation devient absolument impossible.

Rothstein pense que Jérusalem est opposé à la campagne et explique que celui qui n'a pu 'supporter les souffrances à la campagne ne saurait à plus forte raison endurer les peines de la guerre à Jérusalem. Selon P. Volz, Jérémie rencontrerait dans l'avenir une énigme beaucoup plus difficile que celle qu'il se donne aux v. 1 ss. Rudolph est à peu près de cet avis. Weiser

(19) Rudolph, Jeremia 1958, A. Gelin, Jérémie 1951, ad loc. Il en est de même de la monographie la plus récente de J. Leclercq, Les «Confessions de Jérémie» (dans Etudes sur les prophètes d'Israël 1954).

(20) En ce cas, il prendre 11, 18-12, 6 ensemble. 
pense que dans les halliers du Jourdain Jérémie aurait, comme les cavaliers, besoin de plus de courage et d'énergie. ${ }^{(21)}$

Si l'on prend comme nous jusqu'à v. 4 ensemble, on peut penser comme suit: les "piètons" et le "pays tranquille" représentent la situation présente, où la nation existe encore, où Israël, en qualité du peuple élu par Dieu, a eneore le droit de rester à la Terre promise; les "chevaux" et les "halliers du Jourdain" annoncent le temps où la maison de Juda ne sera plus, où toutes les bonnes traditions auront disparu, où Israël, peuple d'alliance, n'existera plus. Dieu n'exige de Jérémie à v. 5 , ni "sacrificium intellectus," ni la "confiance aveugle." $\mathrm{Au}$ lieu de cela, Dieu lui demande de croire toujours à la justice de Dieu, quand le peuple d'alliance s'écroulera, et essaye de l'y préparer. L'idée de la "nouvelle alliance," qui constitue le centre de l'eschatologie de Jérémie se lient donc très logiquement au passage en question. Une autre prophétie de Jérémie le rendra plus clair. Elle concerne la grande sécheresse de 14, 1-15,4. Sans parler ici de la raison pour laquelle j’ai pris ces lignes ensemble, je voudrais entrer tout de suite dans l'essentiel. Jusqu'ici, on pensait: Jérémie prie Dieu à l'occasion de la grande sécheresse pour intercéder auprès de lui pour le peuple, mais Dieu refuse toujours; à travers cette expérience, Jérémie se transforme, d'un prophète de bonheur, en prophète de malheur qui annonce le jugement de Dieu. Cette interprétation est impossible, puisque Jérémie devait s'attendre au jugement dès le moment où il a reçu la vocation. On n'a pas à distinguer ici la prophétie de bonheur d'avec celle de malheur. Il faut voir plutôt en Jérémie le dernier prophète préexilique qui prie sur la foi de l'ancienne alliance. "Nous sommes appelés de ton nom" (9b) montre clairement qu'il s'agit d'Israël; Jérémie prie Dieu de ne pas délaisser ce peuple, s'appuyant sur l'alliance depuis Moïse. “ $\mathrm{Ne}$ profane point le trône de ta gloire. Souviens-toi, ne romps pas ton alliance avec nous" (21) indique bien la situation de Jérémie. "Le trône de ta Gloire" ne veut pas dire temple, mais exprime symboliquement "l'arche de Dieu," c'est-à-dire, l'Amphictytonie israélite. "Ton alliance avec nous" indique l'alliance depuis Moïse, sur la foi de laquelle Jérémie prie Dieu. Mais quand Dieu reste inexorable à cette prière d'intercéssion, Jérémie, forcé de reconnaître les

(21) Rothstein dans H A T, 1922, P. Volz, Der Prophet Jeremia 1928, Weiser, Der Prophet Jeremia $1953-55$ ad loc. 
bornes de l'ancienne alliance, arrive à la nouvelle alliance de 31,31 ss. Celleci n'apparait pas brusquement comme deus ex machina: son apparition est une conséquence nécessaire du caractère logique de la prophétie de Jérémie. L'ancienne alliance, c'est avant tout l'alliance au Sinaï. Chez Jérémie, l'alliance au Sinaï et l'alliance de David coexistaient, mais après sa confrontation avec Joiaqim-décrite au ch. 36-l'alliance de David a été dominée par l'alliance au Sinaï. J'ai parlé ailleurs des circonstances politiques et historiques qui ont suscité cette modification. ${ }^{(22)} \mathrm{Je}$ veux insister seulement ici sur la structure logique de la nouvelle alliance, née de la négation de l'alliance au Sinaï.

Rapprochons la théodicée de Jérémie à celle d'Amos, et nous verrons que chez l'un comme chez l'autre, toute l'argumentation repose sur l'idée d'élection.-N'oublions pas que Jérémie place Israël au centre de sa théodicée.La signification de cette idée deviendra de plus en plus claire, au fur et à mesure que nous traiterons la théodicée des autres historiens israélites.

Je veux parler maintenant du Yahviste. Je dis, que ses travaux se caractérisent par le rationalisme au sens de Weber. Il a transporté beaucoup de traditions cultuelles du plan d'expérience immédiate au plan historique, en les spiritualisant, et en les rationalisant. Je crois voir derrière cette rationalisation le développement de la conscience historique, qui résulte de la formation du royaume et la naissance de la classe intellectuelle qui, privée de pouvoir politique, tourne les yeux critiques vers la réalité du royaume. Ce que le Yahviste met en question, en attitude rationaliste et logique, c'est justement le problème du mal, par conséquent, celui de théodicée. Le Yahviste qui examine de front la source du mal dans Gen. 3, ne fait pas Dieu responsable des péchés, ni des malheurs qui en résultent; ce qui montre bien qu'il était profondément intéressé à la question de théodicée. D'autre part quand il dit: "le serpent était le plus rusé de tous les animaux de champs que Yahvé Dieu avait faits" (Gen. 3,1), il barre le chemin du dualisme. Le mal augmente de plus en plus; même le Deluge ne peut l'ôter. Le Yahviste tâche donc de trouver la solution de ce problème, qui est en même temps celui de théodicée, dans l'histoire du salut, c'est-à-dire, dans la formation de peuples après la destruction de la Tour de Babel et l'élection d'Israël parmi ces peuples. Pour ne pas parler trop longuement, voyons seulement ce que

(22) Sekine, Davidsbund und Sinaibund bei Jeremia (Vetus Testamentum 1959 p. 47 ss) 
dit Neher de cette question. Il voit dans le récit de la Tour de Babel le thème du affrontement de Dieu et de l'homme, et dit que ce thème ne se trouve pas chez les Mésopotamiens, mais chez les Grecs, en particulier dans le mythe de Promethée. Il explique le fait par les mots suivants: "L'univers suméro-babylonien, ne connaît entre les dieux et les hommes que des relations de force et de ruse. Les Hébreux et les Grecs savant qu'un affrontement réel est possible, une lutte dangereuse mais exaltante entre Dieu et l'homme."(23)

Ordinairement nous voyons dans le mythe de Promethée, plus particulièrement dans "Prometheus vinctus" d'Eschyle, la forme typique de la théodicée en Grèce. (24) Mais Neher nous fait voir dans l'histoire de la Tour de Babel aussi une tentative pour la théodicée. Il est clair, si l'on prend les récits du Yahviste dans l'ensemble, que ses efforts pour résoudre la question du mal commencent à partir du moment de la vocation d'Abraham, c'est-à-dire, tout de suite après la destruction de la Tour de Babel. En ce sens, le début de Gen. 12 est très important. Ici comme ailleurs, Israël est opposé aux autre peuples (v. 3 “ toutes les nations de la terre), et l'idée d'élection s'annonce déjà. Nous n'avons pas le temps d'examiner mot par mot les récits du Yahviste ni de parler de la thèse de M. Noth, qui, différente de l'interprétation traditionelle, ne voit pas la conclusion du Yahviste au ch. 1 du Livre des Juges. $^{(25)}$ D'ailleurs cela n'a pas beaucoup d'importance ici, car ce que nous avons prouvé, c'est que la théodicée du Yahviste a pour idée centrale celle d'élection et que la théodicée d'Amos, de Jérémie, et d'autre prophètes part toujours de cette idée. Seulement, le Yahviste ne connaît pas encore, à la différence des prophètes, l'idée de jugement, qui nie l'idée même de l'élection. Le jugement a été certes exécuté par la prise de Jérusalem et la Déportation à Babylone, mais l'idée d'élection subsiste encore dans la foi et la pensée des Israélites et sert de point de départ à leur théodicée. La responsabilité personelle, traitée dans la partie centrale de la théodicée d'Ezechiél, c'est-a-dire, dans le ch. 18, se propose d'avoir un neuveau peuple d'élection, formé de l'initiative de ses membres, c'est-à-dire une communauté religieuse.

Le Code sacerdotal est un grand système de théodicée, conçu pour cette

(23) A. Neher, L'essence du prophétisme 1955, p. 144.

(24) Sekine, Job et Promethée (The Bulletin of the Tokyo Kyoiku University Literature Department for 1960) (en japonais).

(25) Noth, Überlieferungsgeschichtliche Studien I 1943. 
communauté. Il est évident que la Code a une grande part à la théodicée, si l'on lit ce fameux refrain-“et Dieu vit que cela était bon" de Gen. 1. ${ }^{(26)}$ Sans aucun doute le centre du Code est-il le culte, au point de vue pratique, mais au point de vue théorique, c'est l'idée d'alliance. Il faut donc faire attention à l'alliance avec Noé. Au Code sacerdotal la question d'alliance remonte jusqu'a Noé, tandis que le Yahviste ne la fait remonter qu'aussi loin qu'Abraham. C'est cette “ alliance éternelle" qui prévient le jugement de Dieu à l'égard des maux de genre humain.

Nous avons vu que la question de théodicée est toujours étroitement liée à celle de peuple élu, chez le Yahviste, chez les prophètes comme dans le Code sacerdotal. De ce point de vue, la théodicée dans le Livre de Job, que l'on mettait jusqu'ici au centre de la question de la théodicée dans l'Ancien Testament, est au contraire hors du courant principal. Elle ne parle que des souffrances purement personnelles, indépendantes de celles du peuple. Sans aucun doute on ne peut pas niér l'existence d'une théodicée dans Job, puisqu'on y voit agîr puissamment le logos,--au lieu que, dans ce qu'on appelle les “psaumes de théodicée", il n'est question que de l'expérience religieuse vécue par des individus. Ce logos s'occupe de la relation dialectique de la création et du salut, indépendemment des alliances historiques. Nous pensons donc qu'il ne faut pas faire attention à le seule partie des dialogues, mais à la dernière partie qui parle de la révélation de Dieu, c'est-à-dire au ch. $38 \mathrm{ss,}$ qui comporte la dialectique entre la création et le salut. ${ }^{(27)}$

(26) W.F. Albright (Mélanges bibliques rédigée en l'honneur de André Robert 1957, p. 240 ss) propose une nouvelle interprétation de ce refrain. Mais cette interprétation nous doit pas être accepté sans réserve.

(27) Sekine, Schöpfung und Erlösung im Buche Hiob (Festschrift Eissfeldt 1958). 